\title{
CLINICAL USE OF MAGNETIC RESONANCE IMAGING FOR THE DIAGNOSIS OF ACUTE MYOCARDIAL INFARCTION IN THE SURVIVORS OF CARDIAC ARREST
}

\author{
Miroslav Solar̆ ${ }^{1}$, Jan Žižka ${ }^{2}$, Jiři Ceral ${ }^{1}$, Ludovít Klzo ${ }^{2}$ Petr Pařizek ${ }^{1}$
}

Charles University in Prague, Faculty of Medical in Hradec Králové and University Hospital Hradec Králové, Czech Republic: $1^{\text {st }}$ Department of Internal Medicine ${ }^{1}$, Department of Diagnostic Radiology ${ }^{2}$

Summary: The ventricular arrhythmias with underlying coronary artery disease are a leading cause of sudden cardiac death (SCD). While the SCD survivors with proven AMI are considered to be at low risk of SCD recurrence, those without the evidence of AMI represent a high risk group that benefits from implantable cardioverter defibrillator. Therefore, the evaluation of SCD survivors for the presence of acute myocardial infarction (AMI) as a triggering factor of cardiac arrest is essential. In SCD survivors, the use of the standard diagnostic criteria of AMI may be difficult, as both serum cardiac biomarkers and electrocardiogram can be influenced by previous cardiac arrest. A novel technique that may be used for the diagnosis of AMI is magnetic resonance imaging (MRI). We report its use in four patients after cardiopulmonary resuscitation where the diagnosis of AMI could not be definitely established or excluded by means of other diagnostic procedures.

Key words: Magnetic resonance imaging; Sudden death; Myocardial infarction

\section{Introduction}

The most common cause of sudden cardiac death (SCD) is coronary artery disease $(19,21)$ with ventricular tachyarrhythmias as the most frequent underlying mechanism (6). There is a high risk of cardiac arrest recurrence in SCD survivors when the cause of arrhythmia is not reversible $(20,32)$. The majority of resuscitated SCD cases with low risk of recurrence represent the patients that develop malignant arrhythmias in the acute phase of myocardial infarction. Therefore, the precise clinical evaluation for the reversible triggering factors of malignant ventricular arrhythmia, especially acute myocardial infarction (AMI), is important in patients surviving cardiac arrest.

In SCD survivors, the use of the standard diagnostic criteria of AMI (1) may be difficult, as the analysis of pre-arrest symptoms is often impossible and both serum cardiac biomarkers and electrocardiogram (ECG) can be influenced by previous cardiac arrest and cardiopulmonary resuscitation.

A novel technique that may be used for the diagnosis of AMI is magnetic resonance imaging (MRI). We report its use in four patients after SCD where the diagnosis of AMI could not be definitely established or excluded by means of other diagnostic procedures.

\section{Case reports}

Case 1

A 54-year-old man collapsed when traveling by public transport. A by-standing medical student diagnosed circulatory arrest and immediately started basic resuscitation. Advanced cardiac life support was given 5 minutes after the onset of the event. Ventricular fibrillation (VF) was terminated by the second defibrillation shock after administration of $1 \mathrm{mg}$ of adrenaline. Restoration of sinus rhythm led to the rapid return of spontaneous circulation (ROSC). On admission at ICU the patient was comatose, haemodynamically stable and artificially ventilated. ECG showed only mild ST segments depressions $(<0.1 \mathrm{mV})$ in the leads V2-4 that were not apparent after 18 hours. Echocardiography described no regional wall motion abnormality with ejection fraction $(\mathrm{EF})$ of $55 \%$. On admission, there were no laboratory abnormalities explaining the cardiac arrest. Cardiac troponin $\mathrm{T}(\mathrm{cTnT})$ became positive $(1.0 \mathrm{ng} / \mathrm{ml}) 18$ hours after admission.

Mild therapeutic hypothermia was implemented for 24 hours. The patient was extubated 2 days after admission. Confusion and agitation resolved completely in 4 days.

Coronary angiogram performed, after 7 days of hospitalization, showed significant $(80 \%)$ stenosis of the proxi- 
mal left anterior descending coronary artery (LAD). As this finding was inconclusive in regard to the diagnosis of AMI, the cardiac MRI was performed 8 days after the event. Myocardial oedema, accompanied by delayed contrast-enhancement of the subendocardial portion of the anterior wall, confirmed the diagnosis of AMI. When the coronary angiogram was reviewed in the context of the MRI finding, occlusion of the diagonal branch at the site of its separation from LAD was identified as a culprit lesion.

Based on these results, the ICD implantation was not indicated. No cardiac event occurred during the 30 months of follow up.

\section{Case 2}

A 68-year-old man underwent elective PCI of an $80 \%$ ostial stenosis of the right coronary artery (RCA). His medical history was remarkable for non-Q myocardial infarction 2 months before, arterial hypertension, monoclonal gamapathy and chronic dorsalgia.

During stent primomplantation, the patient reported a brief period of chest pain that resolved spontaneously after intracoronary balloon deflation. After the procedure, there were no changes on the ECG tracing. Four hours later, the patient had cardiac arrest with no preceding symptoms. At the time of the event, the patient was on the general ward and his ECG was not monitored. Cardiopulmonary resuscitation was initiated with a short delay. VF was successfully cardioverted with the first defibrillation shock given within 5 minutes after collapse. Because of persistent unconsciousness, the patient was intubated and artificially ventilated.

After CPR, the ECG tracing showed new $2 \mathrm{~mm} \mathrm{ST} \mathrm{seg-}$ ment depressions in the anterior chest leads. Acutely performed coronary angiography showed a good patency of the RCA at the site of the implanted stent and no new

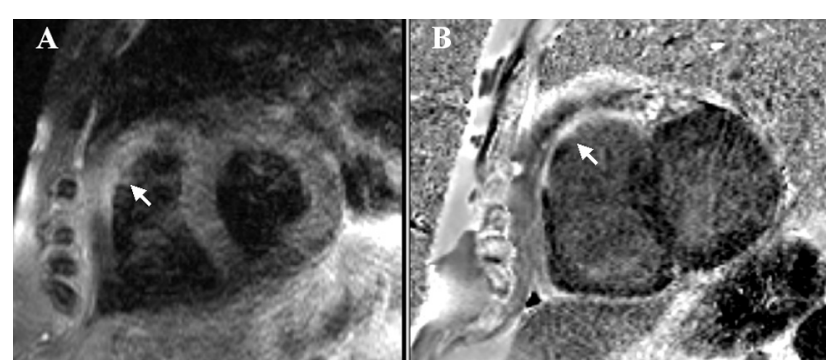

Fig. 1:

A: Fat saturated T2-weighted MRI image, short axis view. T2 - hyperintense myocardial oedema is apparent in the anterior wall of the right ventricle, close to the ventricular outflow tract (arrow).

B: Phase sensitive inversion recovery prepared MR sequence shows late contrast enhancement in the corresponding area of the right ventricular wall, representing the irreversible myocardial injury (arrow). The myocardial infarction developed as a consequence of stent implantation in the proximal RCA. findings in the coronary artery tree. The ST segment changes were not apparent on ECG tracing performed two hours later. cTnT was elevated $(0.5 \mathrm{ng} / \mathrm{ml}) 12$ hours after the event. No serum electrolyte abnormality explaining the ventricular fibrillation was identified.

One day after the event, the patient was extubated with no signs of ischemic brain injury. No arrhythmia occurred during ICU monitoring. Echocardiography performed two days later did not reveal any new findings. Left ventricular function was chronically mildly depressed with EF of $50 \%$ and no regional wall motion abnormalities were found. Although the periprocedural AMI was highly suspected as a causative factor of VF, the available data were not sufficient to make this diagnosis definite. 3 days after the cardiac arrest, a MRI exam was performed. It confirmed the diagnosis of AMI by showing oedema and delayed contrast enhancement of a small portion of the right ventricle supplied by the proximal RCA (Fig. 1). PCI probably led to the occlusion of the small branches stemming from the proximal ACD at the site of the implanted stent. The risk of VF recurrence was thus considered low and the patient was discharged on medical therapy without ICD. The follow up after 8 months was uneventful.

\section{Case 3}

A 53-year-old man with negative medical history had suffered from witnessed cardiac arrest while watching TV. The patient reported no symptoms before the collapse. No basic resuscitation was performed before ambulance arrival. Advanced life support was initiated 6 minutes after the emergency service was called. Ventricular fibrillation was terminated by the second defibrillation shock after administration of $2 \mathrm{mg}$ of adrenaline.

On admission at ICU, the patient was haemodynamically stable, sedated and artificially ventilated. ECG showed mild ST segment elevation $(<0.1 \mathrm{mV})$ in the inferior leads and the tracing did not change in the following hours and days. Acutely performed echocardiography was with normal finding. EF of the left ventricle was $60 \%$ and no wall motion abnormalities were identified. Except for mild cTnT elevation $(0.8 \mathrm{ng} / \mathrm{ml})$, detected 12 hours after admission, blood laboratory analysis revealed no other abnormality.

Mild therapeutic hypothermia was implemented for 24 hours. There were no signs of post hypoxic brain injury when the patient was extubated 2 days after the event.

Coronary angiography revealed three vessel disease but no occlusion or critical narrowing was identified. As the above-mentioned exams did not definitely confirm or exclude the AMI, cardiac MRI was performed 5 days after cardiac arrest. Delayed contrast-enhanced scans revealed a pathological circular subendocardial scar reflecting irreversible myocardial injury. T2-weighted images showed no signs of tissue oedema, typical for an acute myocardial lesion. As AMI was not proved by this method and there was no evidence of myocardial ischemia preceding the cardiac arrest, the ICD implantation was indicated. The device was 
implanted after surgical myocardial revascularization. During the 27 months of follow up, malignant ventricular arrhythmia requiring the ICD therapy did not occur.

\section{Case 4}

A 44-year-old woman collapsed shortly after aerobics training. Her medical history was unremarkable. The collapse was preceded by the symptoms of weakness and malaise. Basic CPR was started 7 minutes after the collapse and the advanced life support was given 5 minutes thereafter. Ventricular fibrillation was terminated by the first defibrillation shock without adrenaline administration with subsequent ROSC. The patient was intubated, artificially ventilated and transferred to the hospital. On admission to ICU, the patient was haemodynamically stable, not requiring circulatory support. On admission, the ECG tracing was normal. A day after the event, the ECG tracing showed terminal negativity of $\mathrm{T}$ waves in the anterior chest leads. Hypokalemia $(2.7 \mathrm{mmol} / \mathrm{l})$ present on admission resolved with unrepeated potassium supplementation and did not recur. Its origin remained unclear. There was no evidence of previous diarrhea or vomiting. Catecholamines were not given during CPR and blood gas analysis revealed normal $\mathrm{pH}$ of 7.35 on admission. Serum cTnT increased to 0.7 $\mathrm{ng} / \mathrm{ml}$ after 12 hours. Echocardiography showed hypokinesis of the cardiac apex and the adjacent septum. The left ventricular EF was $70 \%$.

The patient was extubated 2 days after the event with minor post-hypoxic brain dysfunction. Coronary angiogram, performed 9 days after the event, identified significant stenosis in the periphery of LAD. The rest of the coronary artery tree was normal. As the acuteness of the myocardial lesion was not confirmed by the above mentioned methods and the hypokalemia might have triggered the malignant arrhythmia, cardiac MRI was performed 10 days after the event. It confirmed AMI by showing tissue oedema and subendocardial contrast enhancement in the apex and the apical portion of the interventricular septum. The patient was asymptomatic during the 2 months of follow up.

\section{Discussion}

Clinical diagnosis of AMI is based upon the dynamic changes of serum biomarkers of myocardial necrosis in the context of anginal symptoms, ischemic ECG changes, or coronarographic finding (1). However, there are several pitfalls when these criteria are applied in SCD survivors.

The elevation of serum cardiac biomarkers in patients after CPR may be related not only to AMI but also to the duration of chest compressions, cumulative dose of epinephrine and shock state. $(9,16-18)$ Mild increase of cTnT may also result from the sustained ventricular tachycardia (31) preceding cardiac arrest. The serum concentration of cardiac troponin $\mathrm{T}(\mathrm{cTnT})$ and $\mathrm{MB}$ fraction of creatine kinase lack sufficient specificity for the AMI diagnosis after resuscitated cardiac arrest even when higher than normal cutoff values are used. For a cTnT cutoff value of $0.6 \mathrm{ng} / \mathrm{ml}$ sensitivity and specificity were $96 \%$ and $80 \%$ when AMI was diagnosed by ECG, thallium-201 single-photon emission computed tomography (SPECT) and/or autopsy (16).

The symptoms of angina preceding the cardiac arrest are suggestive of myocardial ischemia but, except for acute coronary syndrome, they may also reflect the ischemia induced by the ventricular tachycardia that preceded cardiac arrest.

Shortly after CPR, the ECG abnormalities may result from previous catecholamine administration, CPR related heart injury and metabolic disturbances related to recent cardiac arrest. ECG interpretation with regard to ongoing ischemia might be especially difficult when there are no significant ST segment deviations or developing changes in the QRS pattern, as it was in all patients described in our report.

Acutely performed echocardiography lacks sufficient specificity and sensitivity to confirm or exclude AMI (10). Small subendocardial lesions are not identifiable by this method. Among our cases, three of four subjects had no regional wall motion abnormality at echocardiography that was performed shortly after cardiac arrest. Regional myocardial dysfunction with preserved wall thickness can be not only a manifestation of AMI, but also an equivalent of myocardial ischemia or old myocardial infarction.

Coronary angiography may be inconclusive for the AMI diagnosis in some cases, especially when the procedure is not performed acutely. Although thrombotic occlusion or critical narrowing of the epicardial coronary artery is a typical feature of AMI, spontaneous or drug facilitated reperfusion can occur. Occlusions of small branches may be difficult to be identified by angiography.

Myocardial perfusion scintigraphy does not allow the differentiation between acute and chronic myocardial infarction, but the acutely necrotic myocardium can be diagnosed using Tc-99m pyrophosphate or indium-111 labeled antimyosin antibody $(13,23)$. The use of this technique was described in 57 SCD survivors with no ECG signs of AMI (12).

A novel technique that can be used in diagnosing AMI is cardiac MRI. Compared to radionuclide imaging, MRI provides significantly better spatial resolution with pixel size of approximately $2 \mathrm{~mm}^{2}$. The paramagnetic contrast agent increasingly accumulates in the areas of both acute myocardial necrosis and fibrotic scar tissue. In an experimental model, the accumulation of paramagnetic contrast agent in the myocardium closely corresponds to both the acute and the chronic myocardial infarction verified by post mortem histological exam $(7,22)$. Contrast-enhanced MRI (CE-MRI) thus provides visualization of irreversible myocardial injury $(11,25)$.

In comparison to a chronic lesion, the AMI is characterized by oedema that develops because of acute tissue necrosis and subsequent inflammatory infiltration. Increased 
water content in oedematous myocardial tissue can be visualized by T2-weighted MR images $(29,30)$.

The combination of contrast-enhanced and T2 weighted MRI enables an accurate differentiation between acute and chronic myocardial infarction. Two studies recently showed high diagnostic accuracy of this method $(3,26)$. Takahashi at al. (27) described the superiority of T2-weighted MRI in comparison to radionuclide imaging for the diagnosis of AMI

The optimal timing of MRI exam in AMI is unclear. The progression of tissue oedema was observed until day 5 after AMI (30) and the oedema was detectable 12 days after the AMI in another report (27). The oedema was not apparent 21 days after AMI (30).

In our study, we used the cardiac MRI imaging protocols described in the literature (3). MRI examinations comprised T2-weighted fast spin echo sequence with fat saturation pulse in order to visualize myocardial oedema and late enhancement inversion recovery prepared gradient echo sequence was used for the assessment of irreversible myocardial injury.

The MRI must be performed before tissue oedema resolves. However, the main limitations of performing cardiac MRI in SCD survivors are post-hypoxic brain injury, respiratory problems and arrhythmias. For cardiac MRI examination, the patient has to be cooperating well and cardiopulmonary compensated. The exam is performed in the supine position using breath hold technique. Breatholds of 7-12 seconds are usually sufficient for adequate image quality on current MR systems.

Although higher long-term risk in AMI complicated by ventricular arrhythmias (4) was reported, observations of both post-MI patients and survivors of cardiac arrest that occurred during the acute phase of transmural MI suggest that life-threatening ventricular tachyarrhythmias occurring during the first 24-48 hours of MI do not imply continuing risk over time $(15,29)$. Current guidelines for the management of ventricular arrhythmias and sudden death do not recommend the implantable cardioverter defibrillator (ICD) in arrhythmias related to acute coronary ischemia (2). On the other hand, ICD therapy proved to be beneficial in the secondary prevention of sudden cardiac death when AMI was excluded as a triggering mechanism of ventricular arrhythmia $(14,28)$.

These facts underscore the importance of precise evaluation of SCD survivors for the presence of AMI. The incorrect AMI diagnosis may cause an undesirable withdrawal from ICD implantation in patients who are at high risk of sudden death recurrence. On the contrary, unrecognized AMI can inadequately increase the costs of treatment and put the patients at risks (5) and limitations (8, 24) related to the ICD implantation.

\section{Conclusion}

Cardiac MRI might be used as an alternative diagnostic tool in SCD survivors in whom the diagnosis of AMI can- not be definitely confirmed or excluded by means of other diagnostic procedures.

\section{References}

1. Myocardial infarction redefined-a consensus document of The Joint European Society of Cardiology/American College of Cardiology Committee for the redefinition of myocardial infarction. Eur Heart J 2000; 21(18):1502-13.

2. ACC/AHA/ESC 2006 Guidelines for Management of Patients With Ventricular Arrhythmias and the Prevention of Sudden Cardiac Death: A Report of the American College of Cardiology/American Heart Association Task Force and the European Society of Cardiology Committee for Practice Guidelines (Writing Committee to Develop Guidelines for Management of Patients With Ventricular Arrhythmias and the Prevention of Sudden Cardiac Death): Developed in Collaboration With the European Heart Rhythm Association and the Heart Rhythm Society. Circulation 2006; 114(10):e385-484. Notes: 10.1161/CIRCULATIONAHA.106.178233

3. Abdel-Aty H, Zagrosek A, Schulz-Menger J et al. Delayed Enhancement and T2weighted Cardiovascular Magnetic Resonance Imaging Differentiate Acute From Chronic Myocardial Infarction. Circulation 2004; 109(20):2411-6. Notes: 10.1161/01.CIR.0000127428.10985.C6

4. Al-Khatib SM, Granger CB, Huang Y et al. Sustained ventricular arrhythmias among patients with acute coronary syndromes with no ST-segment elevation: incidence, predictors, and outcomes. Circulation 2002; 106(3):309-12.

5. Baddour LM, Bettmann MA, Bolger AF et al. Nonvalvular cardiovascular devicerelated infections. Circulation 2003; 108(16):2015-31. Notes: CORPORATE NAME: AHA

6. Bayes de Luna A, Coumel P, Leclercq JF. Ambulatory sudden cardiac death: mechanisms of production of fatal arrhythmia on the basis of data from 157 cases. Am Heart J 1989; 117(1):151-9.

7. Fieno DS, Kim RJ, Chen E-L, Lomasney JW, Klocke FJ, Judd RM. Contrast-enhanced magnetic resonance imaging of myocardium at risk: Distinction between reversible and irreversible injury throughout infarct healing. J Am Coll Cardiol 2000; 36(6):1985-91.

8. Groh WJ, Boschee SA, Engelstein ED et al. Interactions between electronic article surveillance systems and implantable cardioverter-defibrillators. Circulation 1999; 100(4):387-92.

9. Grubb NR, Fox KA, Cawood P. Resuscitation from out-of-hospital cardiac arrest: implications for cardiac enzyme estimation. Resuscitation 1996; 33(1):35-41.

10. Horowitz R, Morganroth J, Parrotto C, Chen C, Soffer J, Pauletto F. Immediate diagnosis of acute myocardial infarction by two-dimensional echocardiography. Circulation 1982; 65(2):323-9.

11. Kim RJ, Fieno DS, Parrish TB et al. Relationship of MRI Delayed Contrast Enhancement to Irreversible Injury, Infarct Age, and Contractile Function. Circulation 1999; 100(19): 1992-2002

12. Krause T, Hohnloser SH, Kasper W, Schumichen C, Reinhardt M, Moser E. Assessment of acute myocardial necrosis after cardiopulmonary resuscitation and cardioversion by means of combined thallium-201/technetium-99m pyrophosphate tomography. Eur J Nucl Med 1995; 22(11):1286-91.

13. Krause T, Zeiher A, Kasper W, Schwehn M, Schumichen C, Moser E. Combined thallium 201/technetium 99m-labeled pyrophosphate tomography for identification of the „culprit“ vessel in acute myocardial infarction. J Nucl Cardiol 1996; 3(2):105-13.

14. Kuck K-H, Cappato R, Siebels J, Ruppel R. Randomized Comparison of Antiarrhythmic Drug Therapy With Implantable Defibrillators in Patients Resuscitated From Cardiac Arrest: The Cardiac Arrest Study Hamburg (CASH). Circulation 2000; 102(7):748-54.

15. Liberthson RR, Nagel EL, Hirschman JC, Nussenfeld SR. Prehospital ventricular defibrillation. Prognosis and follow-up course. N Engl J Med 1974; 291(7): $317-21$.

16. Mullner M, Hirschl MM, Herkner $\mathrm{H}$ et al. Creatine kinase-mb fraction and cardiac troponin $\mathrm{T}$ to diagnose acute myocardial infarction after cardiopulmonary resuscitation. J Am Coll Cardiol 1996; 28(5):1220-5.

17. Mullner M, Oschatz E, Sterz F et al. The influence of chest compressions and external defibrillation on the release of creatine kinase-MB and cardiac troponin $\mathrm{T}$ in patients resuscitated from out-of-hospital cardiac arrest. Resuscitation 1998; 38(2):99-105.

18. Mullner M, Sterz F, Binder M et al. Creatine kinase and creatine kinase-MB release after nontraumatic cardiac arrest. Am J Cardiol 1996; 77(8):581-5.

19. Myerburg RJ. Sudden cardiac death: epidemiology, causes, and mechanisms. Cardiology 1987; 74 Suppl 2:2-9.

20. Myerburg RJ, Kessler KM, Castellanos A. Sudden cardiac death: epidemiology, transient risk, and intervention assessment. Ann Intern Med 1993; 119(12):1187-97. 
21. Pleskot M, Babu A, Kajzr J et al. Characteristics and short-term survival of individuals with out-of-hospital cardiac arrests in the East Bohemian region. Resuscitation 2006; 68(2):209-20

22. Rehwald WG, Fieno DS, Chen EL, Kim RJ, Judd RM. Myocardial magnetic resonance imaging contrast agent concentrations after reversible and irreversible ischemic injury. Circulation 2002; 105(2):224-9.

23. Schoeder H, Topp H, Friedrich M, Jatzkewitz A, Roser M. Thallium and indium antimyosin dual-isotope single-photon emission tomography in acute myocardial infarction to identify patients at further ischaemic risk. Eur J Nucl Med 1994; 21(5):415-22.

24. Sears SF, Todaro JF, Urizar G et al. Assessing the psychosocial impact of the ICD: a national survey of implantable cardioverter defibrillator health care providers. Pacing Clin Electrophysiol 2000; 23(6):939-45.

25. Shan K, Constantine G, Sivananthan M, Flamm SD. Role of Cardiac Magnetic Resonance Imaging in the Assessment of Myocardial Viability. Circulation 2004 109(11):1328-34. Notes: 10.1161/01.CIR.0000120294.67948.E3

26. Stork A, Muellerleile K, Bansmann PM et al. Value of T2-weighted, first-pass an delayed enhancement, and cine CMR to differentiate between acute and chronic myocardial infarction. Eur Radiol 2006.
27. Takahashi N, Inoue T, Oka T et al. Diagnostic use of T2-weighted inversion-recovery magnetic resonance imaging in acute coronary syndromes compared with 99mTc-Pyrophosphate, 123I-BMIPP and 201TICl single photon emission computed tomography. Circ J 2004; 68(11):1023-9.

28. The Antiarrhythmics versus Implantable Defibrillators (AVID) Investigators. A Comparison of Antiarrhythmic-Drug Therapy with Implantable Defibrillators in Patients Resuscitated from Near-Fatal Ventricular Arrhythmias. N Engl J Med 1997; 337(22):1576-84. Notes: 10.1056/NEJM199711273372202

29. Volpi A, Cavalli A, Franzosi MG et al. One-year prognosis of primary ventricular fibrillation complicating acute myocardial infarction. The GISSI (Gruppo Italiano per lo Studio della Streptochinasi nell'Infarto miocardico) investigators. Am J Cardiol 1989; 63(17):1174-8.

30. Wisenberg G, Prato FS, Carroll SE, Turner KL, Marshall T. Serial nuclear magnetic resonance imaging of acute myocardial infarction with and without reperfusion. Am Heart J 1988; 115(3):510-8.

31. Yeo KK, Cruz L, Hong R. Tachycardia-induced elevations in cardiac troponin in the absence of coronary artery disease. Hawaii Med J 2006; 65(3):86-7.

32. Zheng ZJ, Croft JB, Giles WH, Mensah GA. Sudden cardiac death in the United States, 1989 to 1998. Circulation 2001; 104(18):2158-63.

Submitted June 2007. Accepted June 2007.

\section{Corresponding author:}

Miroslav Solař, M.D., University Hospital Hradec Králové, $1^{\text {st }}$ Department of Internal Medicine, Sokolská 581, 50005 Hradec Králové, Czech Republic, e-mail: solarmir@seznam.cz 